\title{
Generación de valor: factor clave en la toma de decisiones de las pymes ${ }^{1}$ Generation of value:
Key factor for the decision-making in the SMEs
}

DOI: http://dx.doi.org/10.17981/econcuc.39.2.2018.01

Artículo de investigación. Fecha de recepción: 15/12/2017. Fecha de aceptación: 02/07/2018

\author{
Nubia Isabel Díaz Ortega \\ Universidad de Pamplona, Pamplona (Colombia) \\ ndiaz712@hotmail.com \\ Marisol Maestre Delgado \\ Universidad de Pamplona, Pamplona (Colombia) \\ mmaestre24@hotmail.com \\ Fernando J. Romero U. \\ Universidad de Pamplona, Pamplona (Colombia) \\ fernadoj57@hotmail.com
}

Para citar este artículo:

Diaz, N., Maestre, M. y Romero, F. (2018). Generación de valor: factor clave en la toma de decisiones de las pymes. Económicas CUC, 39(2). 9-24. DOI: http://dx.doi.org/10.17981/econcuc.39.2.2018.01

\section{Resumen}

La presente investigación tuvo como propósito analizar la influencia de la generación de valor como sustento para la toma de decisiones en las pymes de productos cerámicos de la zona fronteriza colombo-venezolana. Esta es una investigación de tipo correlacional con un enfoque cuantitativo; su diseño fue de campo, no experimental, transaccional. Tuvo una población de 8 gerentes y 8 jefes financieros. Este estudio se fundamenta en los aportes teóricos de García (2009), Knight (2004), Copeland, Koller y Murrín (2004), entre otros. Los resultados muestran una alta influencia de la generación de valor en la toma de decisiones. Como conclusión, las pymes deben fijar acciones para procurar mejorar la generación de valor.

Palabras clave: generación de valor, toma de decisiones, valor económico agregado, gestión financiera. JEL: G19

\section{Abstract}

The purpose of this research was to analyze the influence of value generation as core element for decision making in SMEs of ceramic products located in the border area of Colombia and Venezuela. This is a quantitative study with a correlational non-experimental and transactional approach. It was performed with a population of 8 managers and 8 financial managers. This study is based on the theoretical contributions of García (2009), Knight (2004), Copeland, Koller and Murrin (2004), among others. The results show a high influence of value generation in decision making. In conclusion, SMEs should set actions to try to improve value generation.

Keywords: Value generation, Decision making, Economic value added, Financial management

\footnotetext{
${ }^{1}$ Artículo derivado del proyecto de investigación "Generación de valor como sustento para la toma de decisiones en empresas de productor cerámicos colombo-venezolanas".
} 


\section{Introducción}

Los problemas que se afrontan hoy día, exigen que se mejore la forma de asociación reconstruyendo valores donde los involucrados contribuyan al desarrollo, permitiendo así eliminar la incredulidad sobre los posibles aportes de todos los sectores de la sociedad (Chumaceiro y Hernández, 2016). En este entendido, se presenta la generación de valor representa un tema de interés para las ciencias gerenciales por cuanto involucra el análisis de la complejidad propia de los sistemas empresariales, incluyendo diversidad de elementos del entorno interno y externo en función del logro de los objetivos trazados. Amat (2002) considera que cualquier empresa con ánimo de lucro trata de encontrar dentro de sus objetivos económicos el incremento del valor de la empresa y, por tanto, el de las acciones que integran el capital social la empresa, lo que puede convertirse en un generador de valor o riqueza para sus accionistas.

Bajo el enfoque de la generación de valor, la gerencia de las empresas puede desarrollar las competencias necesarias para ser rentables dentro del entorno apoyado en planes para la solución de problemas y circunstancias experimentadas por las organizaciones. Esta nueva estrategia financiera puede ser considerada a fin de manejar diversos escenarios para una eficaz toma de decisiones.

Por consiguiente, es pertinente realizar estudios enfocados en proporcionar información sobre las variables relativas a la gestión, como es el caso de la generación de valor, así como los aspectos referidos a la toma de decisiones en las distintas organizaciones de interés para los analistas en el área, así lo indica Núñez y Ravina (2017) mencionando que estos estudios están basados fundamentalmente en tres características fundamentales: originalidad, capacidad de adaptación a la realidad y grado de elaboración. En función de ello, el objetivo general del estudio se centró en analizar la generación de valor como sustento de la toma de decisiones en pymes de productos cerámicos de la zona fronteriza colombo-venezolana. Para lograr tales propósitos, se procedió a identificar los elementos claves, la medición del valor y los macroinductores de valor.

Las pymes representan un sector empresarial muy destacado dentro del tejido empresarial colombiano, pues el 99,7\% son catalogadas dentro de este tipo de empresas, (Organización para la Cooperación y el Desarrollo Económicos-OCDE y Comisión Económica para América Latina y el CaribeCEPAL, 2012) y contribuyen al desarrollo económico y social del país. La industria del sector de los productos cerámicos es considerada en Colombia como muy competitivo a nivel internacional, destacándose por su diseño, innovación y tecnología (Dinero, 2015); también recobra importancia por las políticas nacionales propuestas en el Plan Nacional de Desarrollo 2010-2014 (Departamento Nacional de Planeación-DNP, 2010), donde ya fue definida como una de las locomotoras del crecimiento económico.

De igual manera, la importancia del sector de productos cerámicos viene determinada por cifras significativas al 2015, como, por ejemplo, su aporte a nivel de empleo, con 6085 empleos directos; a nivel de impuestos, con un pago de 173.528 millones de pesos; y a nivel del total de ventas de la economía, donde representa un $11 \%$ del total de la industria manufacturera (Asociación Nacional de Empresarios de Colombia-ANDI, 2017).

Tradicionalmente, Norte de Santander cuenta a lo largo de su historia con un sector de empresas dedicadas a la producción cerámica muy importantes (Mogrovejo, Bastos y Pabón, 2015), con aportes al PIB regional por valor de 165.990 millones de pesos que representa el 1,64\% del total del mismo (El Espectador, 2015; Departamento Administrativo Nacional de Estadística-DANE, 2017). Este es un sector exportador que tiene transacciones, principalmente, con países de Centroamérica y Latinoamérica. 
No obstante, varias circunstancias han podido ocasionar una erosión en la situación económica y financiera de todas las pymes del sector cerámico de la zona fronteriza colombo-venezolana, pudiendo generar una iliquidez que impide cubrir todos los pagos, principalmente la prioridad de los salarios de los trabajadores, que es lo más costoso para estas empresas (Romero y Romero, 2016), acompañado del pago a los proveedores con el fin de poder seguir funcionando.

El sector cerámico ha tenido que afrontar problemas del entorno económico nacional pues la economía colombiana ha mostrado el crecimiento más bajo de los últimos tiempos (La República, 2018). Para el DANE, en cifras de febrero de 2017, el Producto Interno Bruto ha descendido desde $4 \%$, en el 2012, hasta llegar a la mitad, en 2016 , con un $2 \%$, y, especialmente, ha golpeado al sector de la construcción, donde la caída ha sido muy fuerte, con cifras que muestran un aporte al PIB del 11,5\% en el 2013 hasta llegar al 4,1\% en el 2016.

Por otra parte, según estudios de la Cámara de Comercio de Cúcuta (2017), la disminución en las ventas, para el sector cerámico, pasaron, desde agosto de 2015, de 7,4 millones USD/FOB hasta $\$ 391.000$ en 2016 , siendo un sector que requiere de una alta inversión en maquinaria, tecnología e insumos importados (Cabedo, Fernández, Marco y Moya, 1999), incidiendo negativamente sobre su rentabilidad (Bastos, 2015). De igual forma, la reforma tributaria del año 2016 fijó el impuesto a la riqueza y aumentó en 3 puntos el IVA a partir del año 2017, lo cual empiezan a sentir las empresas. El peso de la alta carga tributaria se convierte en tasa total de tributación aproximada del $65 \%$, considerada una de las más alta de América Latina (Dinero, 2015), esto impacta la estructura de costos (Domínguez, 2015) y genera tendencias hacia el endeudamiento, además de la pérdida de competitividad en el mercado internacional (Guerrero, 2008).
De todo esto se desprende que, para las pymes de productos cerámicos, en el mediano plazo, es decir, de tres a cinco años, se pudiera presentar déficit de tesorería por la diferencia entre los menores ingresos y costos más elevados: "el desfase existente entre el periodo de conversión del activo corriente y el de exigibilidad del pasivo corriente, requiere un control de gestión" (Pérez, Castro y García, 2009, p. 135). Esta posible iliquidez proporciona una vulnerabilidad y un desequilibrio financiero que se convierte en uno de los factores de la sinistreabilidad de las pymes (Serrano y Callejas, s.f.).

Por tales razones, se demuestra una falta de claridad en la orientación financiera de las pymes de productos cerámicos que les permita generar competitividad (Saavedra, 2012); rentabilidad al fijar sus precios de venta; lograr un mayor aprovechamiento de sus activos fijos; seguimiento y control efectivo a los costos de producción; controlar los niveles de efectivo (Durán, 2011); tomar decisiones considerando el punto de equilibrio; y realizar la adecuada planeación de las utilidades para los nuevos productos (Sánchez, Restrepo y Ospina, 2013).

Entonces, en consonancia con lo planteado, las organizaciones deberían tener claro, cuando toman decisiones, que el aspecto de la generación de valor es vital para la rentabilidad en sus operaciones y para aumentar el flujo de caja, mejorando así su estructura financiera, pues permite ver lo atractivo del crecimiento empresarial (García, 2009). Por ende, a medida que la empresa crece, se mejora la liquidez y su flujo de caja, teniendo oportunidad en sus pagos y gestionar correctamente sus recursos financieros.

El presente documento está organizado en cinco secciones: la primera es esta introducción; la sección segunda revisa la literatura (teoría y evidencia) sobre la generación de valor y la toma de decisiones; la metodología es discutida en la sección tercera; la sección cuarta contiene los resultados; y las conclusiones se presentan en la sección quinta. 


\section{Fundamentos de la generación de valor}

Para el logro de los objetivos que persigue este artículo, sus resultados deben dar un aporte significativo al análisis de generación de valor dentro del proceso de toma de decisiones de las pymes de productos cerámicos, colaborando así, en el largo plazo, al crecimiento sostenido de estas empresas.

Los empresarios deben poseer herramientas adecuadas para establecer una coordinación entre la estrategia general de la organización con sus objetivos financieros, acompañándose de su recurso humano. Estos empresarios deben ser autónomos en la toma de decisiones, tal como Cifuentes, (2013) lo menciona, "la autonomía puede definirse desde varias perspectivas y es así... que se designa como aquella calidad que se predica de quien decide por sí mismo, sin que ello se confunda con el concepto de soberanía o grado máximo de libertad" (p. 315).

En este orden de ideas, la generación de valor se define, según García (2009), como la serie de procesos que conducen al alineamiento de todos los funcionarios con el direccionamiento estratégico de la empresa de forma que cuando tomen decisiones ellas propendan por el permanente mejoramiento de las finanzas a través de la planeación y el control.

Según Sisiruca y Salazar (2014) los valores se encuentran relacionados con el hombre y sus acciones, sin embargo los autores Haddadi, Temeljotov, Foss y Klakegg (2016) expresan que el valor se mira desde varias perspectivas, incluyendo beneficios y utilidades. Este último es un enfoque novedoso para las empresas porque muchas desconocen su existencia, pero al ser aplicado arroja resultados importantes para el éxito de la gestión financiera para medir el crecimiento, fortalecer el patrimonio, y sirven de base para la toma de decisiones (Toro, 2010).

La creación de valor es uno de los retos más importantes para las organizaciones actuales que deben enfrentarlo desde los niveles gerenciales hasta los operativos, es decir, es fundamental lograr que todas y cada una de las personas de la organización entiendan que la relación de sus acciones y decisiones individuales pueden crear o destruir valor según sea el caso. Para Copeland, Koller y Murrín (2004), cuando una empresa haya tomado una decisión clave, necesita comprender los elementos de las actividades diarias, así como qué impacto tienen las decisiones importantes en el valor.

Para Knight (2004), la implementación integral de este concepto puede ser exitosa si las personas con jerarquía son conscientes del sistema (Hernández, Muñoz y Barrios, 2017); por ello, los altos ejecutivos deben tener en cuenta una combinación de aspectos al momento de ejecutar el sistema en función del valor. Este, según Hernández y Chumaceiro (2008), resulta ser un proceso que obliga a promover un desarrollo económico y social en este tipo de organización, sustentado en el avance de esta área, de tal manera que, conocer completamente el programa; darle su respaldo hasta convertirlo en una prioridad para la organización; demostrar su compromiso expresando interés suficiente por los objetivos; y asignar recursos, sobre todo el tiempo de gestión y la energía de las personas, ya que es su responsabilidad directa crear valor para los accionistas, superando las expectativas económicas manifestadas en las acciones de la empresa.

Estos elementos claves de la generación de valor son compromiso de la alta dirección, así como la alineación del proceso de gestión estratégica, la capacitación del personal, la comunicación de resultados y la definición del horizonte temporal, acompañados de la importancia de la medición de los indicadores (Jiménez, 2015) y la fuerza inductora del valor y los macroinductores (MéndezBeltrán y Peralta-Borray, 2014).

De la misma manera, el punto de partida de la generación de valor para una organización es evaluar identificando su fuerza inductora, para lograrlo García (2003) indica que requiere de un sistema de medición 
que permita seguir los resultados obtenidos teniendo en cuenta indicadores como el valor económico agregado, el valor agregado del mercado, la rentabilidad total y la palanca de crecimiento.

Especialmente, García (2003) destaca lo relevante de los macroinductores de valor para comprender como las decisiones afectan el valor de la empresa, es decir, es fundamental el cálculo de la rentabilidad del activo y el flujo de caja libre para identificar y jerarquizar las fuentes de creación y destrucción de valor, dando vía libre para establecer en las pymes de productos cerámicos una fórmula razonable que logre cumplir con su planificación financiera.

\section{Toma de decisiones}

Dentro de los principales temas tratados por los autores en el campo del comportamiento organizacional, se encuentra, con mucha frecuencia, la toma de decisiones como principal foco de acciones empresariales para la aplicación de este concepto. Este tema tuvo una mayor relevancia cuando, en 1978, Hebert Simón ganó el Premio Nobel por su trabajo sobre la toma de decisiones, cambiando la literatura existente que contenía un modelo clásico o meramente racional al proponer uno novedoso basado en el impacto que la conducta humana tiene en las organizaciones, donde cada individuo, grupo y estructura participan racionalmente tomando decisiones sobre opciones de acción más o menos racionales (Simón, 2013).

Por otro lado, Drucker (2006) afirma que la toma de decisiones es la primera habilidad administrativa, así, la mayor parte del éxito de la gestión de los gerentes se deriva de una acertada elección, por tanto, es una de sus principales responsabilidades. Una buena o mala opción afectará negativa o positivamente a la organización, así como también traerá consecuencias sobre la realización de las metas, objetivos y políticas organizacionales. El gerente se dedica a seleccionar un curso de acción en tiempo real sobre innumerables asuntos, como la planeación, ejecución, control y evaluación de resultados.

La toma de decisiones, vista como un proceso, según Herrera (2008), es una serie de pasos que comienza con un número de supuestos hasta concluir con la selección de la mejor alternativa. En la primera parte, se busca definir el problema e identificar los criterios de decisión; en la segunda, asignar prioridades a los criterios, así como desarrollar alternativas; y en la tercera, evaluar las opciones para finalmente hacer la mejor elección.

De la misma manera, Franklin y Krieger (2011) consideran un conjunto de pasos que las organizaciones deben poner en acción para incrementar la probabilidad de que sus elecciones sean tanto lógicas como óptimas. Describe siete puntos a seguir, en los cuales, inicialmente se diagnostica y define la necesidad, el problema o la oportunidad; se establecen metas; se buscan soluciones alternativas, que se comparan, así como se evalúan; para finalmente terminar eligiendo una de ellas. Se implementa entonces la solución seleccionada, y se le da un seguimiento y control a los resultados.

Al mismo tiempo, para Chiavennato (2009), la toma de decisiones es un proceso que consiste en hacer un análisis y elegir, entre varias opciones, un curso de acción. Este proceso implica seis elementos: la persona que toma la decisión, los objetivos, las preferencias, la estrategia, la situación y el resultado. Asimismo, es determinante la percepción del sujeto para explicar su comportamiento en las organizaciones.

Por tal razón, para el autor antes citado, el proceso de toma de decisiones es tan complejo que depende de las características individuales del responsable y lo desarrolla en siete etapas: la percepción de la situación, el análisis, la definición del problema, los objetivos, la búsqueda de opciones de solución, la evaluación, la comparación de opciones, la elección de la alternativa adecuada y la implementación de la misma. 
Los autores antes reseñados concuerdan en afirmar que el proceso de toma de decisiones está compuesto por varias etapas, desde el análisis del problema hasta la solución del mismo con la elección más adecuada. Dependiendo de su punto de vista, Herrera (2008), Franklin y Krieger (2011), Chiavennato (2009) han formulado un conjunto de fases sucesivas que difieren en el número y nombre de los pasos que lo componen.

También, Simón (2013) realizó la clasificación de las decisiones en programables y no programables, resaltando a las primeras por su característica de un grado de estructuración que les permite hacer parte de la rutina de las empresas, mientras las segundas, por el contrario, no pueden incluirse en la rutina porque son situaciones diferentes sin un grado de estructuración. Simón planteó para cada tipo de decisión unos modelos diferentes basados en matemáticas y estadísticas y así darle una respuesta eficaz al problema.

Igualmente, para un alto ejecutivo que diariamente debe tomar decisiones es vital clasificar el tipo o clase de suceso que analiza para, a partir de allí, poder proceder. Según Drucker (2006), no hacerlo constituye un síntoma, consecuencia de un desorden fundamental, aunque también puede tratarse de un suceso aislado. Al realizar una clasificación correcta de la situación, se pueden aplicar distintos procedimientos si se trata de decisiones con suficiente base de información serían descriptivas y se tiene un conocimiento del camino a seguir (García-Peñalvo y Conde, 2016). El tipo de decisión debe concordar con el tipo de acontecimientos que suceden en la organización (Drucker, 2006), el acontecimiento señala el tipo de decisión a tomar.

Por su parte, Franklin y Krieger (2011) indican un tipo de decisión de acuerdo con la magnitud o complejidad de la solución. Teniendo esta base, se pueden adoptar distintos tipos de decisiones que van desde las conocidas y bien definidas hasta las poco comunes e imprecisas, conocidas como controlables e incontrolables (Myskova y Doupalova, 2015).

En razón de lo antes explicado, los diferentes autores de los tipos de decisiones, Drucker (2006), Franklin y Krieger (2011) y Herrera (2008), las clasifican según la magnitud, el número de personas o por la complejidad de la decisión con el objetivo de hacer una correcta evaluación de las diferentes alternativas de solución. Como se pudo observar, todas son posiciones desde diferentes perspectivas, pero, de una u otra manera, complementarias, y por ello deben aplicarse en todos los niveles de la empresa (Ierpetritoy y Sahay, 2016).

Asimismo, Kinicki y Kreitner (2005) muestran la importancia de la toma de decisiones para identificar y elegir entre soluciones alternas que llevan a un estado de cosas deseado, por tanto, es un proceso que inicia con un problema para terminar cuando se ha elegido una solución. Al hablar entonces de toma de decisiones, se hace referencia a la dificultad que se cierne sobre el administrador, la cual consiste en encontrar la decisión adecuada entre varias posibilidades alternas posibles debido a las altas cantidades de información no óptimas a su disposición. Al explorar a otros autores sobre la toma de decisiones, se encuentra a Robbins (2009), quien aplica el concepto como una reacción a un problema, es decir, la diferencia entre un estado actual y un estado deseado se tiene en un problema que le impone una decisión. La calidad de estas decisiones puede verse afectada por la rapidez del mercado actual, exigiéndole al gerente actuar no solamente de una manera efectiva sino hacerlo en un tiempo limitado.

\section{Metododología}

De acuerdo con Lerma (2009), este estudio, según las características de las variables generación de valor y toma de decisiones, se puede catalogar dentro del enfoque cuantitativo. La investigación cuantitativa se 
caracteriza por plantear hipótesis que pueden presentarse como proposiciones que pueden fácilmente ser convertidas con fórmulas matemáticas que expresan relaciones funcionales entre variables, además utiliza técnicas estadísticas estructuradas para el análisis de la información.

Según el nivel de profundidad con el que se pretende abordar el problema de estudio, la presente investigación es de tipo correlacional, como bien lo advierten Hernández, Fernández-Collado y Baptista (2014), porque busca conocer la relación existente entre dos o más conceptos, categorías o variables de modo particular. Es decir, se mide cada variable presuntamente relacionada para medir y analizar su comportamiento de manera correlacionada.

De acuerdo con Bernal (2010), la definición del diseño de investigación está determinada por el enfoque cuantitativo y por la hipótesis que va a probarse durante el desarrollo de la investigación. En atención al diseño esta investigación se clasifica en: no experimental, transeccional- descriptivo, de campo, los cuales se describen a continuación.

Con respecto a esto, para Ortega y Pick (2011) se refiere a las características de cierto grupo de personas o a cómo se desarrolla un fenómeno para buscar conclusiones válidas para esa población. En virtud de lo señalado, a efectos de la presente investigación, se consideraron las pequeñas empresas, clasificadas por la ley 590 del 2000, como aquellas unidades económicas con activos de 501 a 5000 salarios mínimos mensuales y que tengan de 11 a 50 empleos; $\mathrm{y}$, las medianas, con activos entre 500130000 salarios mínimos y que tengan entre 50 y 200 empleados, constituidas legalmente como sociedades mercantiles destinadas, en este caso, a la producción de productos cerámicos de la zona fronteriza colombovenezolana, que estuvieran asociadas en Induarcillas, que realizaran actividades de comercio exterior y que contaran con permisología del Servicio Nacional Integrado de
Administración Aduanera y Tributaria-SENIAT y la Dirección de Impuestos y Aduanas Nacionales-DIAN. De esta manera, el grupo quedó conformado por ocho gerentes y ocho jefes financieros de igual número de pymes del sector cerámico de la zona fronteriza colombo-venezolana.

En cuanto a la población, cuando está completamente definida es conocida como finita, según Bernal (2010), tal como sucede en este caso, donde se conoce el número total de la población; así mismo, la población es accesible puesto que es una porción del universo en estudio al que realmente tiene acceso la investigadora. Ahora bien, dada la población por el número de unidades que la integran es accesible en su totalidad, es decir, no se hace necesario extraer una muestra (Tabla 1).

TABLA 1

Distribución de la población

\begin{tabular}{ccc}
\hline Empresas & Gerentes & $\begin{array}{c}\text { Jefes } \\
\text { financieros }\end{array}$ \\
\hline Gres de los Andes C.A. & 1 & 1 \\
\hline Dist. Multigres C.A. & 1 & 1 \\
\hline Rustigres C.A. & 1 & 1 \\
\hline Tejar de Pescadero S.A & 1 & 1 \\
\hline Ladrillera Merkagres & 1 & 1 \\
\hline Ladrillera Casablanca & 1 & 1 \\
\hline Tejar Santa Teresa & 1 & 1 \\
\hline Cerámica Andina & 1 & 1 \\
\hline
\end{tabular}

Fuente: elaboración propia.

\section{Resultados}

En primer lugar, se elaboró el análisis inferencial. En esta fase de la investigación aparecen los resultados de confiabilidad con un resultado del índice de Alfa de Combrach de 0,929 para la variable generación de valor y de 0,924 para la variable toma de decisiones. Se puede notar que las variables se encuentran en los rangos aceptados y el cuestionario en general alcanza un 0,956 . 
Para corroborar la asociación entre las dos variables se aplicó el coeficiente de Sperman-Browm con un resultado del 0,851 y las dos mitades de Guttman con un 0,849. Como se observa, la hipótesis acepta los tres criterios, por lo que se afirma que la generación de valor influye en la toma de decisiones.

En segundo lugar, se realizó un análisis descriptivo. Al analizar los resultados generales de la variable generación de valor, se obtiene una moderada valoración por parte de los gerentes y jefes financieros de las pymes de productos cerámicos de la zona fronteriza. Es importante establecer que los resultados fueron moderados a nivel de varios indicadores (Tabla 2).

TABLA 2

Dimensión de los elementos claves de la generación de valor de los gerentes y jefes financieros.

\begin{tabular}{ccc}
\hline $\begin{array}{c}\text { Elementos claves de } \\
\text { generación de valor }\end{array}$ & Gerentes & $\begin{array}{c}\text { Jefes } \\
\text { financieros }\end{array}$ \\
\hline $\mathrm{N}$ & 9 & 9 \\
Media & 3,66 & 3,26 \\
Moda & 4 & 3 \\
Desv. típica & 1,041 & 1,205 \\
Mín. & 2 & 1 \\
\hline
\end{tabular}

Fuente: elaboración propia.

A nivel de la dimensión correspondiente a los elementos claves de la generación de valor para gerentes, se obtuvo que el promedio fue de 3,66 , lo cual le ubica en el nivel alto; en función del instrumento, se ubica en la respuesta algunas veces lo cual es favorable. La moda de 4 indica que el valor más frecuente corresponde al nivel alto. La desviación estándar de 1,041 implica baja dispersión de los puntajes alrededor de la media, observándose que el valor mínimo presentado por la muestra fue de 2 y el máximo de 5 .

Por otra parte, se visualiza para los jefes financieros un promedio de 3,26, lo cual le ubica en el nivel moderado. La moda de 3 indica que el valor más frecuente corresponde al nivel moderado $\mathrm{y}$, en función del instrumento, se ubica la respuesta en $a \lg u$ nas veces, indicando que es favorable según el baremo. La desviación estándar de 1,205 implica baja dispersión de los puntajes alrededor de la media, observándose que el valor mínimo presentado por la muestra fue de $1 \mathrm{y}$ el máximo de 5 .

En relación con los resultados, estos reflejan que los elementos claves de la generación de valor son considerados en nivel alto por parte de los gerentes y moderado en los jefes financieros dentro de la actividad realizada por las empresas del sector de productos cerámicos, siendo relevante por cuanto, pese a existir limitaciones, estas organizaciones cuentan con un conjunto de elementos que contribuyen en su creación de valor. Amat (2009) acota que la creación de valor es necesaria para que la empresa logre un crecimiento equilibrado, no solo para obtener beneficios y liquidez sino para crecer de manera sólida.

TABLA 3

Indicadores de los elementos claves de la generación de valor de los gerentes.

\begin{tabular}{cccccc}
\hline & Compromiso & Alineación & Capacitación & Resultados & Horizonte \\
\hline $\mathrm{N}$ & 9 & 9 & 9 & 9 & 9 \\
Media & 4,05 & 3,67 & 3,73 & 3,05 & 3,78 \\
Moda & 5 & 4 & 3 & 3 & 4 \\
Desv. & 0,807 & 1,258 & 1,034 & 1,074 & 1,033 \\
típica & & 2 & 2 & 2 & 2 \\
Mín. & 3 & 5 & 5 & 4 & 5 \\
Max. & 5 & & & & \\
\hline
\end{tabular}

Fuente: elaboración propia. 
En la tabla 3 se muestran los resultados de los indicadores y de la dimensión elementos claves de la generación de valor para los gerentes. En tal sentido, se pone en evidencia que, al medir el indicador compromiso de la alta dirección para los gerentes, el promedio fue de 4,05, lo cual le ubica en el nivel muy alto. La moda de 4 refleja que el valor más frecuente corresponde al nivel alto. La desviación estándar de 0,807 implica una baja dispersión de los puntajes alrededor de la media, observándose que el valor mínimo presentado por la muestra fue de 3 y el máximo de 5 .

En cuanto a la opinión de los jefes financieros, se pone en evidencia que, al medir el indicador compromiso de la alta dirección, el promedio fue de 3,22 , lo cual le ubica en el nivel moderado. La moda de 3 refleja que el valor más frecuente corresponde al nivel moderado. La desviación estándar de 1,093 implica una baja dispersión de los puntajes alrededor de la media, observándose que el valor mínimo presentado por la muestra fue de 1 y el máximo de 5 .

Se señala en función de lo expuesto que para los gerentes predominó un muy alto y para los jefes financieros un nivel moderado en el elemento compromiso de la alta dirección, lo cual demuestra que la misma identifica diferentes perspectivas perseguidas por los encuestados, esto es, los objetivos de la alta gerencia consideran la generación de valor mientras los jefes financieros solo lo hacen algunas veces en las empresas del sector de productos cerámicos de la zona fronteriza colombovenezolana.

Respecto a la alineación del proceso de gestión estratégica para los gerentes, el promedio fue de 3,67 , lo cual le ubica en el nivel alto. La moda de 5 muestra que el valor más frecuente corresponde al nivel muy alto. La desviación estándar de 1,258 implica baja dispersión de los puntajes alrededor de la media, observándose que el valor mínimo presentado por la muestra fue de 2 y el máximo de 5 .

Para los jefes financieros, el promedio fue de 3,38 , lo cual le ubica en el nivel moderado. La moda de 3 muestra que el valor más frecuente corresponde al nivel moderado. La desviación estándar de 1,230 implica baja dispersión de los puntajes alrededor de la media, observándose que el valor mínimo presentado por la muestra fue de 1 y el máximo de 5 .

Desde la perspectiva de los resultados presentados, alto para los gerentes y moderado para los jefes financieros, se denota que la alineación estratégica ha sido realizada por los primeros sujetos en concordancia con el objetivo de crear valor y, para los segundos, algunas veces.

TABLA 4

Indicadores de los elementos claves de la generación de valor de los jefes financieros.

\begin{tabular}{cccccc}
\hline & Compromiso & Alineación & Capacitación & Resultados & Horizonte \\
\hline $\mathrm{N}$ & 9 & 9 & 9 & 9 & 9 \\
Media & 3,22 & 3,38 & 3,17 & 3,33 & 3,22 \\
Moda & 3 & 3 & 3 & 3 & 3 \\
Desv. Típica & 1,093 & 1,230 & 1,029 & 1,327 & 1,348 \\
Mín. & 1 & 1 & 1 & 1 & 1 \\
Max. & 5 & 5 & 5 & 4 & 4 \\
\hline
\end{tabular}

Fuente: elaboración propia. 
Falta conectar el direccionamiento estratégico con la fuerza inductora de valor; lo cual no es favorable para los fines de la generación de valor de las empresas de productos cerámicos. En concordancia con los resultados a que llegó en su investigación Leal (2009), en las empresas del sector eléctrico, uno de los factores relevantes en la organización es el liderazgo que la misma posee, y como primer factor se encuentra el capital intelectual, que influye en la generación de valor económico financiero en las empresas eléctricas facilitando la operatividad y factibilidad de la organización. En igual forma, resultados similares obtuvo Geizelez (2008) en su tesis doctoral sobre empresas del sector carbonífero al obtener en este indicador un nivel adecuado, recomendando establecer las medidas necesarias para la implantación del enfoque de generación de valor a través de un proceso de gestión estratégica formalizado, sistematizado y conocido por todos los actores relacionados con este tipo de empresas.

En cuanto al indicador capacitación del personal para los gerentes, el promedio fue de 3,73 , lo cual le ubica en el nivel alto. La moda de 5 refleja que el valor más frecuente corresponde al nivel muy alto. La desviación estándar de 1,034 implica baja dispersión de los puntajes alrededor de la media, observándose que el valor mínimo presentado por la muestra fue de $2 \mathrm{y}$ el máximo de 5 .

Sin embargo, para los jefes financieros, el promedio fue de 3,17 , lo cual le ubica en el nivel moderado. La moda de 3 refleja que el valor más frecuente corresponde al nivel moderado. La desviación estándar de 1,029 implica baja dispersión de los puntajes alrededor de la media, observándose que el valor mínimo presentado por la muestra fue de 1 y el máximo de 5 .

A efecto de los resultados expuestos, se denota que para los gerentes es muy alto el nivel reflejado con respecto a la capacitación, mientras que para los jefes financieros es moderado, lo cual implica que existe una diversidad de criterios en la formulación clara del programa de educación, capacitación y las actividades complementarias en el programa de creación de valor; no tienen en cuenta a todo el personal, siendo ello un poco desfavorable para las empresas de productos cerámicos. De acuerdo con González (2010), quien llegó a una similitud de resultados en su investigación doctoral sobre empresas consultoras de software, se denota un nivel moderado en este indicador, mostrando una debilidad en estas empresas, ya que la capacitación es un instrumento mediante el cual los miembros de la organización interiorizan la manera de crear valor.

Dichos resultados son contradictorios con los planteamientos de García (2003) quien sostiene que el proceso de capacitación del personal en la permanente creación de valor debe enfocarse en tres importantes aspectos para generar interés en el programa: comprender la importancia de los conceptos de valor, integrarlos a las decisiones diarias y diseñar un sistema de comunicación.

En lo que respecta al indicador comunicación de resultados para los gerentes, el promedio fue de 3,05, lo cual le ubica en el nivel moderado. La moda de 3 significa que el valor más frecuente corresponde al nivel moderado. La desviación estándar de 1,074 implica baja dispersión de los puntajes alrededor de la media, observándose que el valor mínimo presentado por la muestra fue de 2 y el máximo de 5 .

En cuanto a la opinión de los jefes financieros, el promedio fue de 3,33 , lo cual le ubica en el nivel moderado. La moda de 3 significa que el valor más frecuente corresponde al nivel moderado. La desviación estándar de 1,327 implica baja dispersión de los puntajes alrededor de la media, observándose que el valor mínimo presentado por la muestra fue de 1 y el máximo de 5 . 
En atención a los resultados mostrados, se muestra un nivel moderado para los gerentes y de igual manera para los jefes financieros con respecto a la comunicación de resultados, denotando, según los encuestados, que se realizan ciertas acciones vinculadas con la comunicación de resultados del programa de generación de valor, no obstante, deben realizarse actividades comunicativas para que produzcan efectos positivos en las empresas de productos cerámicos.

Según los planteamientos de Knight (2004), un programa exitoso de comunicación debe estar basado en la repetición, refuerzo, recepción y redundancia para que le permitan lograr la comprensión necesaria a las personas y, así, incorporar el lenguaje del valor.

De igual forma, los resultados tampoco coinciden con lo expresado por Copeland, Koller y Murrín (2004), quienes sustentan, como base para cumplir con la generación de valor, que este elemento es importante, ya que contribuye y apoya el logro de los objetivos de la organización. Representa la mejor forma para comprobar periódicamente los logros obtenidos a través de foros informativos que incluyen análisis y revisiones de rendimiento.

En referencia con el indicador definición del horizonte temporal para los gerentes, su promedio fue de 3,78 , lo cual le ubica en el nivel alto. La moda de 4 muestra que el valor más frecuente corresponde al nivel alto. La desviación estándar de 1,033 implica baja dispersión de los puntajes alrededor de la media, observándose que el valor mínimo presentado por la muestra fue de 2 y el máximo de 5 .

Para los jefes financieros, se pone en evidencia un promedio de 3,22 , lo cual le ubica en el nivel moderado. La moda de 3 muestra que el valor más frecuente corresponde al nivel alto. La desviación estándar de 1,327 implica baja dispersión de los puntajes alrededor de la me- dia, observándose que el valor mínimo presentado por la muestra fue de $1 \mathrm{y}$ el máximo de 5 .

Los datos obtenidos reflejan que para los gerentes se obtuvo un nivel alto y los jefes financieros moderado, lo que permite inferir que en las empresas de productos cerámicos existe una diferencia de criterios en la definición del horizonte temporal, en analizar el número de años requerido para determinar los logros del objetivo crear valor y efectuar estudios permanentes para saber cuánto tiempo necesitan los ejecutivos para coordinar las expectativas de valor a alcanzar, siendo ello no tan favorable para las empresas de productos cerámicos. Las evidencias anteriores tienen relación con los aportes teóricos de Copeland, Koller y Murrín (2004), quienes afirman que el primer paso para establecer la generación de valor lo constituyen el número de años que va a cubrir la propuesta y su nivel de detalle para tener un impacto positivo en el futuro de la empresa si se aprovecha este tiempo de forma oportuna y adecuada, implicando las variables decisivas y reales que podrían generar muy altos desempeños.

A nivel de la variable toma de decisiones, los resultados de las etapas de este tipo de proceso se presentan en la Tabla 5.

TABLA 5

Dimensión de las etapas del proceso toma de decisiones para los gerentes y jefes financieros.

\begin{tabular}{ccc}
\hline $\begin{array}{c}\text { Etapas proceso } \\
\text { toma de decisiones }\end{array}$ & Gerentes & $\begin{array}{c}\text { Jefes } \\
\text { financieros }\end{array}$ \\
\hline $\mathrm{N}$ & 9 & 9 \\
Media & 3,83 & 3,46 \\
Moda & 4 & 3 \\
Desv. Típ. & 0,994 & 1,035 \\
Mín. & 1 & 1 \\
Max. & 5 & 5 \\
\hline
\end{tabular}

Fuente: elaboración propia. 
En la dimensión etapas del proceso de toma de decisiones para gerentes, se obtuvo que el promedio fue de 3,83 , lo cual le ubica en el nivel alto y en las respuestas algunas veces según el instrumento confeccionado para esta investigación. La moda de 4 significa que el valor más frecuente corresponde al nivel alto. La desviación estándar de 0,994 implica baja dispersión de los puntajes alrededor de la media, observándose que el valor mínimo presentado por la muestra fue de 1 y el máximo de 5 .

Sobre las respuestas de los jefes financieros, estas arrojaron un promedio de 3,45, lo cual le ubica en el nivel alto. La moda de 3 significa que el valor más frecuente corresponde al nivel moderado. La desviación estándar de 1,035 implica baja dispersión de los puntajes alrededor de la media, observándose que el valor mínimo presentado por la muestra fue de 1 y el máximo de 5 .

Vinculando el análisis de los resultados obtenidos en esta investigación para la dimensión etapas del proceso de toma de decisiones con los mostrados por los antecedentes, se puede señalar que en varias investigaciones las diversas etapas como factor clave del proceso decisorio en las organizaciones se examinaron y explicaron bajo diferentes puntos de vista, como Salazar (2011) en el sector pymes, Labarca (2010) en los comandos del Ejército Bolivariano, Sabina (2010) en empresas manufactureras, Oviedo (2010) en universidades públicas, e, indistintamente del tipo de empresa, se detectó la importancia de las etapas continuas y sucesivas del proceso de toma de decisiones para la gestión en cada una de ellas.

Atendiendo a los resultados obtenidos, ubicados en un nivel alto, se demuestra que las empresas de productos cerámicos conocen las etapas del proceso de toma de decisiones, los cuales son favorables para esta gestión, siendo ello importante para los fines de contar con aspectos que contribuyen con la efectividad de sus operaciones.

En relación con el análisis de la generación de valor como sustento para la toma de decisiones en las pymes de productos cerámicos, se puede indicar que este proceso puede configurarse como sustento para el establecimiento del enfoque de generación de valor como un sistema holístico que permite tomar decisiones gerenciales más eficientes, por cuanto los gerentes y el área financiera de estas organizaciones pueden desarrollar conocimientos que sirvan para el desarrollo de sus funciones, siendo el compromiso de la alta dirección uno de los elementos claves, desde la perspectiva de Knight (2004), quien señala que está relacionado con la viabilidad del programa de creación de valor.

\section{Conclusiones}

El objetivo de la presente investigación fue determinar el grado de influencia de la generación de valor en la toma de decisiones en las pequeñas y medianas empresas del sector de productos cerámicos, por lo que se pudo obtener que la generación de valor juega un papel primordial en la toma de decisiones, por ende, es importante para las pymes fortalecer y reforzar su programa de creación de valor.

En la investigación se caracterizaron los elementos claves de la generación de valor desarrolladas por las pymes de productos cerámicos, encontrándose que el nivel de compromiso de la alta dirección fue alto, indicando que contribuye con el horizonte perseguido por las empresas; también se encontró que es moderado con respecto a la alineación del proceso de gestión estratégica, la cual ha sido enunciada de una manera no tan adecuada con los objetivos organizacionales. Así mismo, se precisó lo moderado del nivel en cuanto a la capacitación del personal, no identificándose plenamente con los resultados de la generación de valor.

Igualmente, se describieron las etapas del proceso de toma de decisiones desarrolladas por las pymes de productos cerámicos, y se señaló que la definición del problema la realizan en un nivel moderado; y, el establecimiento de metas, la búsqueda de soluciones alternativas, la evaluación de so- 
luciones y la elección entre soluciones alternativas se da en un nivel alto. En cuanto a la implementación de la solución seleccionada, el nivel obtenido fue de moderado, y, por su parte, el control de resultados fue alto, lo cual implica que se formulan las mismas en correspondencia con los objetivos.

A la gerencia general se le recomienda seguir fomentando el compromiso de las pymes de productos cerámicos con la generación de valor a través de la actualización de las políticas en esta materia, así como por medio de la realización de seminarios y actividades internas para promover los valores empresariales que incluyan la generación de valor a fin de procurar un mayor fortalecimiento de la misma.

Asimismo, la investigación llena un vacío en la literatura existente sobre la influencia de la generación de valor en la toma de decisiones y su relevancia se enmarca al profundizar en un tema de interés para los ámbitos empresariales y académicos de la región, pues con el conocimiento de los resultados se posibilita el planteamiento de soluciones concretas al problema expuesto, y estas tienen una gran importancia desde el punto de vista económico, ya que el sector cerámico es representativo por su gran aporte al desarrollo económico de la región.

Dado que el estudio se concentró en las pymes de productos cerámicos de la zona fronteriza, los resultados, así como las conclusiones que de ellos se derivan, pueden no ser extrapolables a otros contextos. Así pues, el artículo ofrece datos que pueden ser útiles para otras empresas a efectos de conocer la importancia de la generación de valor en la toma de decisiones, pero deben ser usados con la precaución de considerar las diferencias de contexto que puedan llegar a surgir.

Por último, el trabajo presenta oportunidades para líneas de futuras investigaciones pues se cuenta con dos vertientes: i) la necesidad de que las pymes repiensen su alineación estratégica en cuanto a la generación de valor, y ii) el análisis de las causas del porqué la generación de valor no contribuye óptimamente a la toma de decisiones.

\section{Referencias}

ANDI. (2017). La industria cerámica en Colombia. Informe Comité Sector Cerámico. [En línea]. Consultado el: 1 de abril de 2018. Disponible en http:// www.andi.com.co/Uploads/LaIndustriaCeramicaDeColombia.pdf

Amat, O. (2002). Valor Económico Agregado. Barcelona: Ediciones Gestión 2000.

Amat, O. (2009). Análisis integral de empresas. Claves para un chequeo completo: desde el análisis cualitativo al análisis de balances con casos prácticos. México, D.F.: Alfaomega.

Bastos, L. (2015). La paridad cambiaria dólar/peso en la liquidez y rentabilidad de las MiPyME del sector de confecciones en la ciudad de Cúcuta, años 20052008. Universidad \& Empresa, 14(22), 151-167

Bernal, C. (2010). Metodología de la investigación. Administración, economía, humanidades y ciencias sociales. Bogotá, D.C.: Pearson.

Cabedo, J. Fernández, M., Marco, M. y Moya, I. (1999). La estructura financiera del sector de pavimentos y revestimientos cerámicos. Revista de treball, economía $i$ societat, 15, 35-42.

Cámara de Comercio de Cúcuta. (2017). Panorama Económico $2016 \quad y$ proyecciones 2017. Recuperado de http:// www.cccucuta.org.co/media/Adjuntos de_Noticias/informe_economico.pdf

Chiavenato, I. (2009). Comportamiento Organizacional. La dinámica del éxito de las organizaciones. México, D.F.: Mc Graw Hill.

Chumaceiro, A. y Hernández, J. (2016). Países generadores de políticas públicas para elfomentodelacultura empresarial dirigida hacia la responsabilidad social. Revista Telos, 18(1). 103-120. Disponible en http://ojs.urbe.edu/index.php/telos/ article/view/790 
Cifuentes, G. (2013). Desarrollo Constitucional, Legal y Jurisprudencia del Principio de Rigor Subsidiario. Jurídicas CUC, 9(1), 345 - 384.

Copeland, T. Koller, T. y Murrín, J. (2004). Valoración, medición y gestión del valor. Barcelona: Deusto.

DANE. (2017). Producto Interno Bruto. Consultado el 1 de abril de 2018. Disponible en http://www.dane.gov.co/index.php/52-espanol/noticias/ noticias/4383-producto-interno-brutopib-oferta-iii-trimestre-2017

Departamento Nacional de Planeación, DNP. (2010). Plan de Desarrollo Nacional: 'Prosperidad para todos' 20102014. Bogotá, D.C.: DNP.

Dinero. (2015). Impacto profundo. Disponible en http://www.dinero.com/edicion-impresa/caratula/articulo/ impacto-del-impuesto-riqueza-empresas-colombia/208506

Domínguez, J. (2015). An Analysis of the Technological Structure of Refineries and Blenders Estimation of the Leontief Multiproduct Cost Function and Reservation Prices. Equidad \& Desarrollo, 23, 147-186 Recuperado de https:// dialnet.unirioja.es/descarga/articulo/5166573.pdf

Drucker, P (2006). La toma de decisiones. Bogotá, D.C.: Deusto.

Durán, Y. (2011). Administración del capital de trabajo: una herramienta financiera para la gerencia de las Pymes tradicionales venezolanas. Visión Gerencial, 1, 37-56.

El Espectador. (2015). Cúcuta diversifica su economía ante el declive del comercio con Venezuela. Consultado el 28 de marzo de 2018. Disponible en https:// www.elespectador.com/noticias/economia/cucuta-diversifica-su-economia-eldeclive-del-comercio-articulo-541511
Franklin, E. y Krieger, M. (2011). Comportamiento Organizacional. Enfoque para América Latina. México, D.F.: Editorial Pearson. Prentice Hall.

Hernández, J. y Chumaceiro, A. (2008). Sociedad del conocimiento y pertinencia social universitaria. CEISEP una propuesta para la UNERMB. Revista Venezolana de Ciencias Sociales, UNERMB. 12(1). 77-92.

Hernández, R., Fernández-Collado, C. y Baptista, P. (2014). Metodología de la

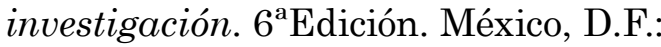
Mc Graw-Hill.

Ierpetritoy, M. y Sahay, N. (2016). Decision Making Across Different Scales: From Process Control to Supply Chain Management. Computer Aided Chemical Engeneering, 38, 2397-2402.

García, O. (2003). Valoración de empresas. Gerencia de Valor y EVA. Medellín: Digital Express.

García, O. (2009). Administración financiera. Fundamentos y aplicaciones. Cali: Prensa Moderna.

García-Peñalvo, F. y Conde, M. (2016). Using informal learning for business decision making and knowledge management. Journal of Business Research, 67(5), 686-691.

Geizelez, T. (2008). Gestión estratégica como fundamento para la generación de valor en las empresas del sector carbonífero del estado Zulia. [Tesis]. URBE, Maracaibo.

González, K. (2010). Capital relacional y generación de valor en empresas consultoras del software del sector petrolero. [Tesis]. Universidad Rafael Belloso Chacín, Maracaibo.

Guerrero, J. (2008). Mecanismos fiscales para la globalización económica y social. Un enfoque de hacienda pública internacional. Economía industrial, 370, 33-43. 
Haddadi, A., Temeljotov, A., Foss, M. y Klakegg, O. (2016). The Concept of Value for Owners and Users of Buildings - A Literature Study of Value in Different Contexts. Procedia - Social and Behavioral Sciences, 226(1). 381389.

Hernández, H., Muñoz, D. y Barrios, I. (2017). Estilos gerenciales y su influencia en la generación de valor de las instituciones prestadora de salud de la región caribe. Económicas CUC, 38(1), 133-146.

Herrera, M. (2008). Toma de decisiones en ambientes turbulentos. Modelos y herramientas para las ciencias de la complejidad. México, D.F.: Universidad Autónoma de Baja California.

Jiménez, J. (2015). El inductor-PDC enfocado a medir el crecimiento y generación de valor para la PYMES. Dimensión Empresarial, 13(1). 151165.

Kinicki, A y Kreitner R, (2005). Comportamiento organizacional, conceptos, problemas y prácticas. México D.C.: Mc Graw Hill.

Knight, J. (2004). La administración integral basada en el valor. Desarrollo de una aproximación sistemática a la creación de valor para el accionista. Bogotá, D.C.: Mc Graw-Hill.

La Republica (febrero 15, 2018). La economía colombiana registro el peor crecimiento de los últimos ocho años. Disponible en : https://www.larepublica.co/economia/la-economia-colombiana-registro-el-peor-crecimiento-de-losultimos-ocho-anos-2599653

Labarca, R. (2011). Inteligencia emocional como herramienta gerencial para la toma de decisiones de los directores del comando general del Ejército $\mathrm{Na}$ cional Bolivariano. CIGAC, 7(2), 63 80
Leal, C. (2009). Capital intelectual y generación de valor económico financiero en empresas del sector eléctrico. [Tesis]. Universidad Rafael Belloso Chacín, Maracaibo.

Lerma, H. (2009). Metodología de la investigación. Propuesta, anteproyecto y proyecto. Bogotá, D.C.: ECOE.

Méndez-Beltrán, J. y Peralta-Borray, D. (2014). Reflexiones respecto a la responsabilidad social empresarial y la creación de valor económico desde la perspectiva de los proveedores. Cuadernos de Contabilidad, 15(38), 625-645. Disponible en https://oi.org/10.11144/ Javeriana.cc15-38.rrrs

Mogrovejo, J., Bastos, L. y Antuny, J. (2015). Impacto económico del sector cerámico en San José de Cúcuta (Colombia). Universidad \& Empresa, 17(29), 157-180.

Myskova, R. y Doupalova, V. (2015). Approach to risk management decisionmaking in the small business. Journal Procedia and Finance, 34, 329-336.

Núñez, E. y Ravina, R. (2017). Análisis del nivel de competitividad empresarial en el panorama publicitario colombiano fundamentada en el éxito creativo y la responsabilidad ética y jurídica. Jurídicas CUC, 13(1). 9-28. http://dx.doi.org/10.17981/juridcuc.13.1.2017.1

OCDE y CEPAL. (2012). Perspectivas económicas de América Latina 2013: políticas de pymes para el cambio estructural. $\mathrm{Pa}-$ ris: OCDE. Disponible en https://reposit o r i o.cepa l.org / b itstrea m / handle/11362/1463/1/S2012083_es.pdf

Ortega, I. y Pick, S. (2011). Investigación en ciencias sociales: paso a paso. México, D.C.: Limusa.

Oviedo, M. (2010). Coaching gerencial como herramienta para el proceso de toma de decisiones. [Tesis]. Universidad Rafael Belloso Chacín, Maracaibo. 
Pérez, J., Castro, O. y García, B. (2009). Gestión del capital circulante y rentabilidad en las pymes. Revista Contabilidad y Dirección, 9, 11-140.

República de Colombia. Congreso de la República. (2 de agosto de 2004). Promoción del desarrollo de los micros pequeñas y medianas empresas. [Ley 590]. DO: 45.628.

Romero, Y. y Romero, Y. (2016). Identificación de efectos económicos-comerciales. La ciudad fronteriza de Cúcuta a causa de la crisis colombovenezolana del 2015. [Tesis]. Esumer, Medellín.

Robbins, S. (2009). Comportamiento organizacional. México D.C.: Pearson Prentice Hall.

Saavedra, L. (2012). Una propuesta para la competitividad de la Pyme latinoamericana. Pensamiento y Gestión, 33(1), 93-124.

Sabina, C. (2010). Gestión del riesgo financiero como herramienta para la toma de decisiones gerenciales del sector manufacturero. [Tesis]. Universidad Rafael Belloso Chacín, Maracaibo.

Salazar, R. (2011). Diagnóstico del proceso de toma de decisiones para la selección de proyectos de inversión en pequeñas y medianas empresas metalmecánicas. CIGAC, 8(1), 84-94.

Sánchez, J. Restrepo, F. y Ospina, H. (2013). La planeación financiera un modelo de gestión en las mypymes. Face, 13(1), 137-150. https://:doi. org/10.24054/01204211.v1.n1.2013.964

Serrano, D. y Callejas, M. (s.f.). Causas de la siniestralidad de las MiPymes en Colombia: Factores internos. [Tesis de maestría]. EAFIT, Medellín.

Simón, H. (2013). Administrative Behavior. A study of decision-making processes in administrative organization. $4 \mathrm{ed}$. New York: The Free Press.
Sisiruca, M. y Salazar, C. (2014). Valores éticos de la responsabilidad social interna en centros de producción audiovisual. Económicas CUC, 35(1), 79-90.

Toro, D. (2010). Análisis financiero: enfoque proyecciones financieras. Bogotá, D.C.: ECOE.

Nubia Isabel Díaz Ortega es Docente de la Facultad de Ciencias Económicas y Empresariales, Universidad de Pamplona, Pamplona - Colombia. Contador público con Postdoctorado en Ciencias de las Organizaciones.

Marisol Maestre Delgado es docente de la facultad de Ciencias Económicas y Empresariales, Universidad de Pamplona, Pamplona - Colombia. Economista con Magíster en Educación.

Fernando J. Romero U. es docente titular de Postgrados de la Universidad de Zulia, Maracaibo - Venezuela. Doctor en Ciencias Gerenciales. 